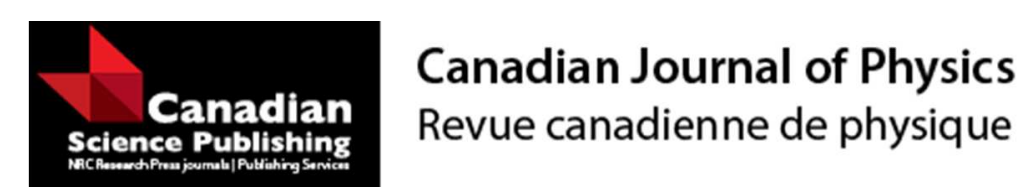

\title{
Atypical thermal transport in Cu nanorods in the diffusive- ballistic crossover
}

\begin{tabular}{|r|l|}
\hline Journal: & Canadian Journal of Physics \\
\hline Manuscript ID & cjp-2015-0469.R1 \\
\hline Manuscript Type: & Article \\
\hline Date Submitted by the Author: & 18 -Apr-2016 \\
\hline Complete List of Authors: & $\begin{array}{l}\text { Karmakar, P.K.; Tezpur University, Physics } \\
\text { Mohanta, D; Tezpur University, Physics }\end{array}$ \\
\hline Keyword: & Nanorod, Metallic system, Thermal transport, Hot-spot, Heat-flow equation \\
\hline
\end{tabular}




\title{
Atypical thermal transport in $\mathrm{Cu}$ nanorods in the diffusive-ballistic crossover
}

\author{
P K Karmakar*, ${ }^{*}$ and D Mohanta ${ }^{2}$ \\ Department of Physics, Tezpur University, Napaam-784028, Tezpur, Assam, INDIA. \\ *Corresponding author E-mail: ${ }^{1}$ pkk@tezu.ernet.in; ${ }^{2}$ best@tezu.ernet.in
}

\begin{abstract}
We propose a simple theoretical calculation scheme based on the phenomenological Fourier heat-flow formalism to study thermal transport behaviors in nanoscale copper rods. The axial heat transport is characterized by a new super-oscillatory feature along with small-amplitude heat-spikes. It is anticipated that, these atypical spikes are generated by accumulation of localized "hot-spots" that have low-heat dissipation characteristics. In case of radial transport, we witness the existence of three distinct heat-regimes owing to buildup of hot electrons after experiencing ballistic scattering events. As an important surveillance, even though the nanorod diameter is comparable or smaller than the electron mean free path length, $\lambda_{m f p} \sim 30 \mathrm{~nm}$; multiple ballistic electronic scattering from the outer surface of the nanorods and subsequent accrual into several layers through secondary collisional events has led to concentric heat-zones. The hot-spots disappear when the diameter exceeds $\lambda_{m f p}$.
\end{abstract}

Keywords: Nanorod; metallic system; thermal transport; hot-spot

\section{Introduction}

In recent years, thermal properties of low-dimensional systems have gathered immense interest due to fundamental concern and relevance in diverse areas of nanotechnology research $[1,2]$. An abrupt flow of heat in metallic and semiconductor nanostructures can lead to generation of hot-spots, where heat is believed to be confined spatially around the voids and defects. In a typical semiconductor, the existence of transient hot-spots can prevail up to a time-scale larger than the phonon relaxation time $\left(\sim 10^{-11} s\right)$. The heat dissipation raises critical issues in a number of components including heat sinks, thermo-photovoltaics, quantum contacts and junctions [1-4]. Quantum junctions that act as the foundation behind every quantum device and quantum architecture are usually fabricated through sophisticated tools, like nanolithography and focused ion beam techniques [5-8]. In these miniaturized devices, heat dissipation at the interconnects and interfaces that make use of nanowires and nanorods, pose serious problems while considering guided current flow with absolute precision. This is because, surface scattering of electrons, adsorption of impurity atoms from the ambient environment, high sensitivity to thermal fluctuations etc. cannot be ignored in structures of reduced dimension. For instance, at elevated temperatures plasmonic response could be hindered due to impurity scattering as well as carrier scattering [9].

On the other hand, magnetic field-induced heat dissipation is extremely beneficial in the treatment of hyperthermia [10,11]. As far as bioengineering and biomedical research are concerned, nano-dimensional metallic systems have widely been employed as anti-bacterial and anti-microbial agents [11,12]. More so, metallic powders are in practice in making implants in bone tissues and tooth cavities $[13,14]$. Recently, nanotubes and nano-whiskers have been considered as a vital mode of channels in diagnostics and therapeutics including targeted drug delivery [15]. Although there have been reports [16-19] as regards, heat generation, conduction, and dissipation, however, there is a long standing demand to 
understand transport phenomena in one-dimensional (1D) structures using evolutionary model equations for metallic nanorods that form the basis of nano-electronic components. It may again be noted that electrons are the major contributor to heat transport for metals at large, whereas semiconductors, owing to lack of ample free electrons, support thermal response by the way of lattice vibrations through excitation of phonons [16,17].

\section{Mathematical formalism}

Herein, we formulate phenomenological heat-flow dynamics of regular copper $(\mathrm{Cu})$ nanorods possessing an axial symmetry. The independent axial and radial contributors to heat transport are as worked out below. In our formulation, the modified heat-diffusion equation $[18,19]$ along the $z$-direction (axial), with thermal diffusivity $h$ and temperature distribution $T$ in coordination space $(z, t)$ is given as,

$$
\frac{1}{h} \frac{\partial T}{\partial t}=\frac{\partial^{2} T}{\partial z^{2}},
$$

with all the other symbols having their usual meanings. The normalized (by standard $\mathrm{Cu}$ parameters) form of equation (1) can be written as,

$$
\frac{\partial \Theta}{\partial \tau}=\alpha \frac{\partial^{2} \Theta}{\partial z^{2}}
$$

where, the standard normalization scheme adopted in our investigation is described as,

$$
\Theta=\frac{T}{T_{m p}}, \tau=\frac{t}{\tau_{e}}, Z=\frac{z}{\lambda_{m f p}} \text { and } \alpha=\frac{h \tau_{e}}{\lambda^{2}{ }_{m p}} \text {. }
$$

Here, $T_{m p}$ represents the melting point $(=1358 K)$ temperature of Copper, $\tau_{e}$ stands for the electron relaxation time scale $\left(\sim 2 \times 10^{-14} s\right)$, and $\lambda_{m f p}$ signifies the electronic mean free path $(\sim 30 \mathrm{~nm})$ [16-17]. Similarly, the radial form of equation (1) to analyze the evolutionary kinetics of the radial heat-flow (long $r$-direction) can be expressed as,

$$
\frac{1}{h} \frac{\partial T}{\partial t}=\frac{\partial^{2} T}{\partial r^{2}}+\frac{1}{r} \frac{\partial T}{\partial r} \text {. }
$$

The normalized form of equation (3) in normalized radial direction $\left(\xi=r / \lambda_{m f p}\right)$ is given by,

$$
\frac{\partial \Theta}{\partial T}=\alpha\left(\frac{\partial^{2} \Theta}{\partial \xi^{2}}+\frac{1}{\xi} \frac{\partial \Theta}{\partial \xi}\right) \text {. }
$$

We shall computationally employ the modified Fourier formalism, described by derived phenomenological equations (2) and (4), for exploiting heat transport behavior in some technologically relevant $\mathrm{Cu}$-based situations.

\section{Results and discussions}

We numerically integrate the heat-flow governing equations [Eqs. (2) \& (4)] under the usual boundary conditions [16-21] that the temperature and its gradient are extremum at the initial and boundary points. The initial values are self-explanatory axis-wise in the obtained patterns reflected in Figs. 1-3. We apply the fourth-order Runge-Kutta method for purely spatial profiles and the finite-element method for purely spatiotemporal patterns. Figure 1 depicts a profile of axial heat transport through a nanoscale solid metallic cylinder of diametric size 20 $\mathrm{nm}$ with an axial length normalized to $\lambda_{m f p}(=30 \mathrm{~nm})$ and temperature normalized to $T_{m p}$ of $\mathrm{Cu}$ $(=1358 \mathrm{~K})$. As can be found, temperature grows rapidly and attains a value close to melting 
point at a length scale comparable to $\lambda_{m f p}$. The broad peak maxima can be ascribed to electronic temperature of the most scattered electrons in the vicinity of the axial center. The temperature does not drop rapidly beyond the central point due to sustained collisional events beyond $\lambda_{m f p}$. At the end of the rod under study, however, an elevated temperature reaching the melting point regime is realized due to the proximity effect. The effect can be ascribed to the successive electron collisions from the end terminal face via ballistic scattering. Note that, ballistic scattering occurs at the end-surfaces without any collisional event occurring along the carrier path. From Fig. 1, it can be noticed that the end terminal of the nanorods has experienced an abrupt rise in temperature beyond a normalized scale length of 2.25.

The spatiotemporal profile of the axial heat transport is illustrated in Fig. 2. The plot is made using normal Fourier heat flow equation and considering normalized length, normalized temperature as well as time normalized over the electron relaxation time $\left(\sim 2 \times 10^{-14} \mathrm{~s}\right)$. Basically, it depicts a super-oscillatory (oscillation within oscillation, OWO) behavior of temperature variation with progressive length and elapsed time. The thermal waves, with continuously varying amplitudes, travel while accommodating small-scale amplitude waves. A group of thermal wavelets are expected to evolve nonlinearly at near the extreme end of the cylindrical structure. Interestingly, a series of curves that experience peak maxima at a normalized temperature of 0.5 and length scale of unity decays steadily with the elapsed time. The steady fall of amplitude of the thermal wave leads to some kind of damped waveform followed by a flat behavior until a normalized length of 1.5. Beyond this critical value, the spikiness is shifted to a lower degree and is attributed to proximity effect. The proximity effect is clearly characterized by localized waves at the edge boundary and normalized time scale of unity (indicated by red encircled region). The existence of OWOs is basically a result of thermal waves mediated by large density of electrons along with the thermal waves owing to generation of localized hotspots.

In addition to the axial feature, we have also intended to exploit heat conduction mechanism with consideration of the radial part. Fig. 3 depicts evolutionary temperature with variation in radius in normalized form. As can be found, initially the solid cylinder has experienced an abrupt temperature rise as one goes along from the axial center to a normalized radial length of 0.05 . Consequently, a steep rise can be witnessed beyond this value along with a peak maximum obtained at 0.18 . Furthermore, we have noticed broad, subsidiary thermal peaks corresponding to normalized lengths of $\sim 0.34$ and 0.47 . The existence of isolated peaks implies formation of hot-spots in concentric radial shells along the radially outward direction from the principal center (Fig.4). It has been anticipated that, an adequate surface scattering of electrons from the outer boundaries followed by accumulation into definite radial zones would lead to a system of different concentric heat-zones. Since the electrons in $\mathrm{Cu}$ give an approximate $\lambda_{m f p}=30 \mathrm{~nm}$, it is possible that multiple scattering from the outer surfaces followed by redistribution into various radial zones would lead to a transient rise of temperature as compared with that in other segments. The hot-spots are thus created out of accumulated electron scattering in a confined geometry that results in various zones. The radial cross-sectional view of thermal transport is shown in Fig. 4. It basically highlights the nature of ballistic scattering [19-21] of the electrons contributing to the development of hot-spot regimes. The solid (A), dashed (B) and dotted (C) line-based arrows correspond to three independent trajectories of the electrons participating in forming hot-spot regimes after encountering respective ballistic scattering events. It may be noted that, in case of metals, a major part of the thermal transport dynamics is governed by the electrons, unlike lattice vibrations in semiconductors. We can say that, any nanorod having diameter smaller, or comparable to the $\lambda_{m f p}$-dimension is likely to experience ballistic scattering of the carriers. It is possible that a sufficiently large number of electrons pile up into different zones upon 
multiple scattering from different regions of the walls, yielding uniformly distributed hot-spot elements. Correspondingly, we notice three broad peak maxima in the normalized radial temperature profile. Although, the diameter of the nanorods is comparable or smaller than $\lambda_{m f p}$, multiple ballistic scattering from the outer surface of the rod can lead to concentric heat zones as a secondary collisional effect. The zones are nothing but accumulation of closely spaced hot-spots distributed within a layer from the zone center. More particularly, there exist three hot-spots of gradually falling strengths corresponding to diametric scales of 14.7, 27.4 and $37.9 \mathrm{nms}$, respectively. The hot-spots regions are more significant for the first two cases, but offers a smearing away trend for the third case (Fig. 3-4) In a regime beyond such diametric lengths $\left(>>\lambda_{m f p}\right)$, the hot-spots disappear after some kind of local squeezing effect of the super-oscillatory nature.

\section{Conclusions}

We have demonstrated hot-spot mediated thermal transport of copper nanorods in the diffusive-ballistic regime [19-21]. The accumulation of localized hot-spots that have low-heat dissipation characteristics can be ascertained from the spatiotemporal profiles numerically generated from the modified phenomenological heat-flow formalism. In the radial transport, even though the diameter of the nanorods is smaller than $\lambda_{m f p}$, multiple ballistic scattering from the outer surface of the rod followed by secondary collisional events would lead to concentric heat zones. Moreover, the hot-spots smear out when the diameter of the nanorods surpass $\lambda_{m f p}$.

It may be worth mentioning that, since the electron mean free path length is larger than the typical diameter of the nanorods under study, the analytic formulation meant for radial heat-flow is manifested by ballistic scattering of electrons. Conversely, the generic unidirectional heat-flow is not affected along the axial direction. In this backdrop, the spatial double-derivative in the phenomenological Fourier heat-flow formalism would not diverge abruptly throughout the model nanosystem under study.

In addition to the above, the nanorods under examination are believed to be free from voids and imperfections, and possess continuous lattice structure. If the nanorods, in contrast, contain certain amount of defect density, then $\lambda_{m f p}$ and $\tau_{e}$ both will be altered. But, the alteration is more significant in response to temperature variation $\left(\lambda_{m f p} \sim T^{-0.5}\right)$ than the inclusion of defects [16-17]. Moreover, surface defects are only prominent in the case of nanostructures that possess the defects available at the core only.

The methodological analyses presented here, despite the above facts and faults, would find immense scope in a number of diverse technologically relevant $1 D$ systems, such as, nanorods, nanobelts, nanopillars, nanotubes, etc. with additional corrections required due to the inclusion of defects and impurities.

Acknowledgements: Authors duly thank their colleagues for fruitful discussions. The contribution of the anonymous learned Referees, via insightful comments and constructive suggestions, is gratefully recognized. 


\section{References}

1. E. T. Swartz, R. O. Pohl, Rev. Mod. Phys. 61, 605 (1989).

2. D. G. Cahill, W. K. Ford, K. E. Goodson, G. D. Mahan, A. Majumdar, H. J. Maris, J. Appl. Phys. 93, 793 (2003).

3. H. Houten, L. W. Molenkamp, C. W. J. Beenakkar, C. T. Foxon, Semicond. Sci. Technol. 7, B215 (1992).

4. M. A. Sierra, D. Sanchez, Phys. Rev. B 90, 115313 (2014).

5. S. Y. Chou, P. R. Krauss, P. J. Renstrom, Science 272, 85 (1996).

6. M. J. Madou, Fundamentals of Microfabrication: The Science of Miniaturization $\left(2^{\text {nd }}\right.$ ed., CRC, 2002).

7. J. Orloff, M. Utlaut, L. Swanson, High Resolution Focused Ion Beams: FIB and Its Applications (Kluwer Academic/Plenum Publishers, USA, 2003).

8. A. Jordan, R. Scholz, P. Wust, H. Fähling, R. Felix, J. Magn. Magn. Mater. 201, 413 (1999).

9. A.L. Lereu, R. H. Farahi, L. Tetard, S. Enoch, T. Thundat, A. Passian, Optics Exp. 21, 12145 (2013).

10. R. Xu, Y. Zhang, M. Ma, J. Xia, J. Liu, Q. Guo, N. Gu, IEEE Trans. Magnetics 43 (3) 1078 (2007).

11. S. Dutz, R. Hergt, Nanotechnology 25, 452001 (2014).

12. A. Gupta, S. Silver, Nat. Biotechnol. 16, 888 (1998).

13. J. R. Morones, J.L. Elechiguerra, A. Camacho, K. Holt, J. B. Kouri, J. T. Ramırez, M. J. Yacaman, Nanotechnology 16, 2346 (2005).

14. C. V. S. Subba Rao, P. P. Vanajasan, V. S. Chandana, Trends Biomater. Artif. Organs. 25, 75 (2011).

15. S. J. Ahn, S. J. Lee, J. K. Kook, B. S. Lim, Dent. Mater. 25, 206 (2009).

16. G. Burns, Solid State Physics (Academic Press, Inc., New York, 1985).

17. C. Kittel, Introduction to Solid State Physics ( $8^{\text {th }}$ ed., Wiley, 2005).

18. T. N. Narasimhan, Revs. Geophys. 37, 151 (1999).

19. D. G. Cahill et al., Appl. Phys. Revs. 1, 011305 (2014).

20. P. E. Hopkins, P. M. Norris, J. Heat Transfer, 131, 022402 (2009)

21. R. Gebarowski, P. Seba, K. Zyczkowski, J. Zakrzewski, Euro. Phy. J. B 6, 399 (1998).

\section{Figure captions}

Fig. 1 Profile of axial heat transport through a nanoscale solid metallic cylinder of diametric size $20 \mathrm{~nm}$ with an axial length normalized to electron mean free path length $(40 \mathrm{~nm})$ and temperature normalized to melting temperature $(1358 \mathrm{~K})$.

Fig. 2 Spatiotemporal profile of axial heat transport through a nanoscale solid metallic cylinder with various inputs and normalizations described in the text. The proximity effect is clearly characterized by localized waves at the edge boundary and normalized time scale of unity (indicated by red encircled region).

Fig. 3 Profile of radial heat transport through a nanoscale solid metallic cylinder with various inputs and normalizations described in the text.

Fig. 4 Schematic representation for radial cross-sectional view of thermal transport mediated by ballistic scattering of the electrons resulting in hot-spots. Here, solid (A), dashed (B) and dotted (C) arrow-based lines correspond to three independent trajectories of the electrons participating in forming the hot-spot regimes after encountering ballistic scattering, respectively. 


\section{Figures}

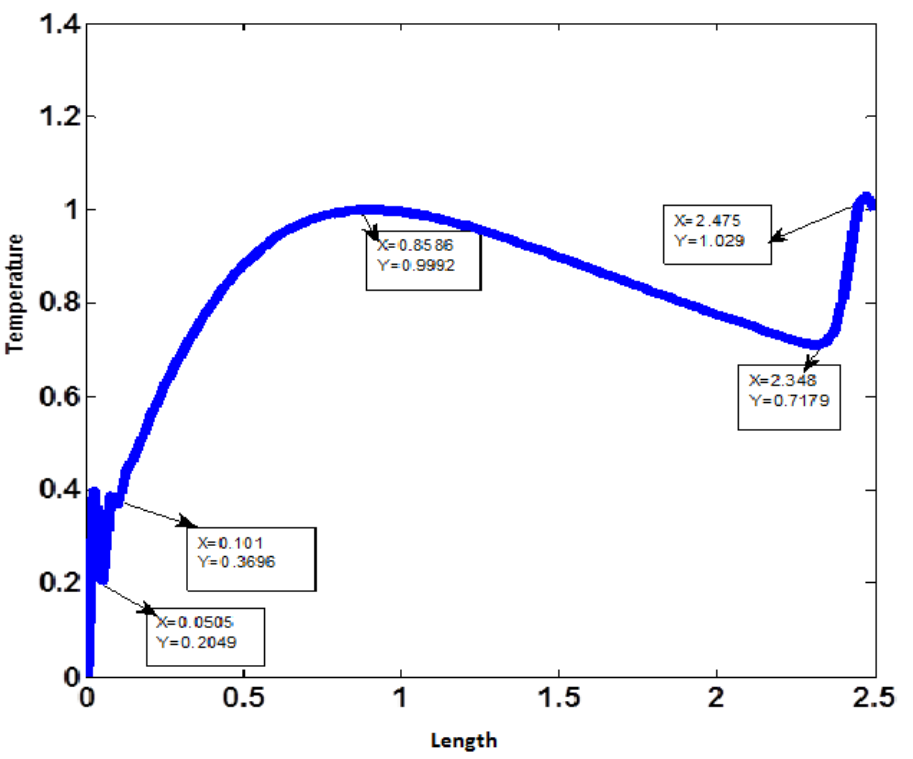

Fig. 1.

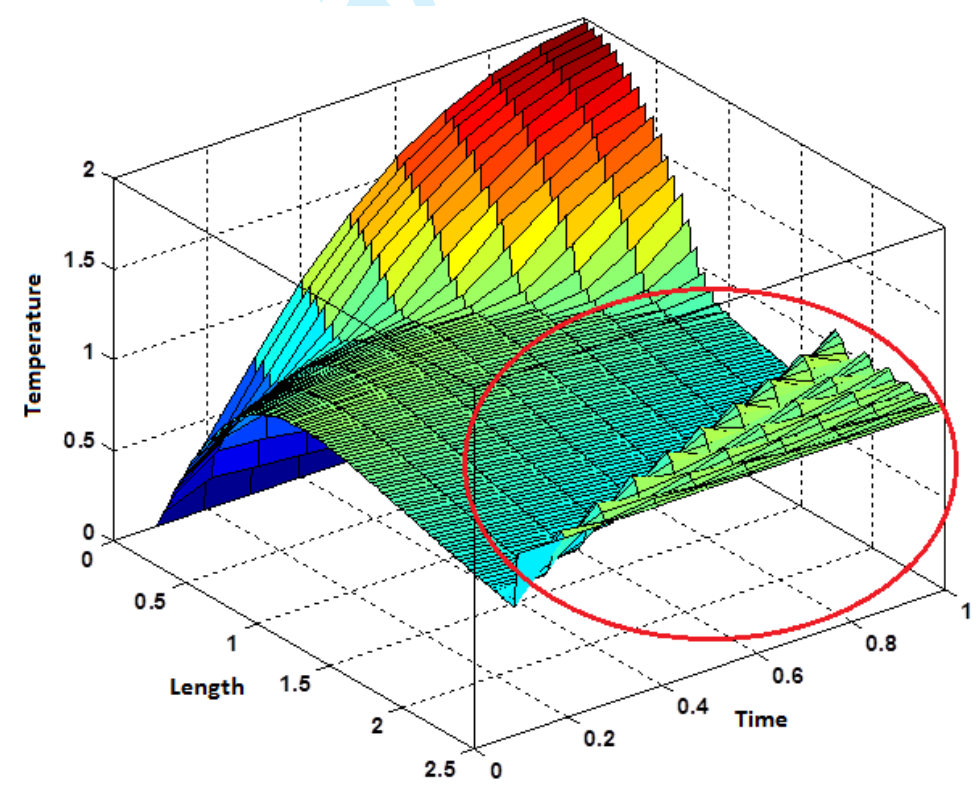

Fig. 2 




Fig. 3.

Fig. 4.

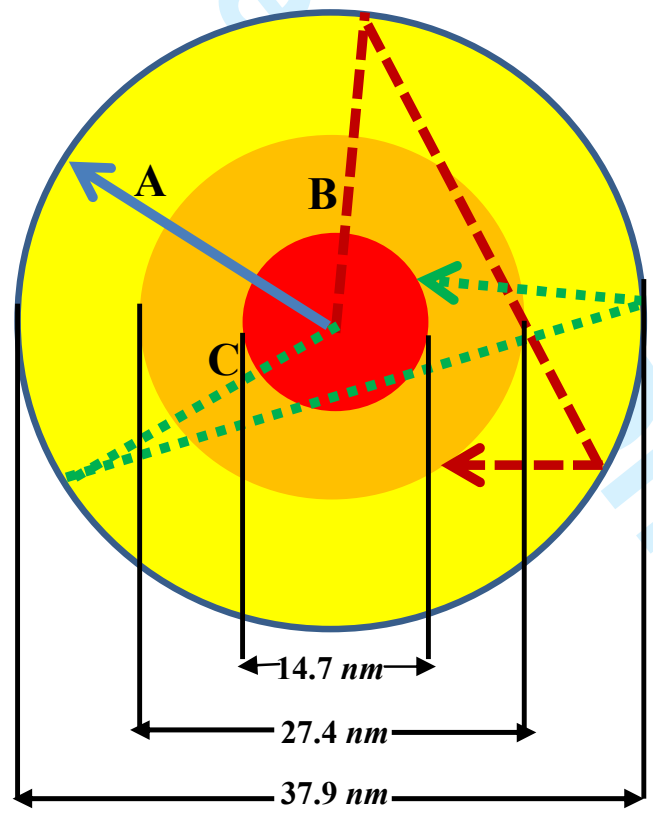

